\title{
Unusual isolated extramedullary relapse of acute lymphoblastic leukemia in the breast despite complete donor hematopoietic chimerism after allogeneic hematopoietic stem cell transplantation
}

Pack Hyun Sung, Changjo Im, Shin Young Hyun, Kwang Yong Shim, Jong In Lee, and Jee Hyun Kong

Division of Hematology-Oncology, Department of Medicine, Wonju Severance Christian Hospital, Yonsei University Wonju College of Medicine, Wonju, Korea

Received: April 6, 2015

Revised : June 16, 2015

Accepted: July 16, 2015

\section{Correspondence to}

Jee Hyun Kong, M.D.

Division of Hematology-Oncology,

Department of Medicine, Wonju

Severance Christian Hospital,

Yonsei University Wonju College

of Medicine, 20 Ilsan-ro,

Wonju 26426, Korea

Tel: +82-33-741-1201

Fax: +82-33-731-5884

E-mail: kkongg@yonsei.ac.kr
To the Editor,

Allogeneic hematopoietic stem cell transplantation (HSCT) is an established treatment for high-risk acute leukemia. The curative effect of allogeneic HSCT for acute leukemia is attributed to the graft-versus-leukemia effect produced by allogeneic immune cells, as well as intensive conditioning chemotherapy with or without radiotherapy. However, relapse remains the major cause of treatment failure after allogeneic HSCT for acute leukemia.

Relapse monitoring for acute leukemia after allogeneic HSCT is typically performed by chimerism analysis, which characterizes the hematopoietic stem cell origin of the recipient's blood or bone marrow after allogeneic HSCT. Increasing mixed chimerism is a sign of hematological relapse [1]. Chimerism analysis predicts not only bone marrow relapse, but also extramedullary relapse (EMR). In a retrospective study of 146 adults with acute lymphoblastic leukemia (ALL) who underwent allogeneic HSCT, all isolated EMR patients exhibited either increased mixed chimerism in the peripheral blood only, or in both the bone marrow and the blood before relapse [1]. However, we recently experienced a case of an
ALL patient with isolated EMR of the breast, despite complete donor chimerism.

The patient, a 32-year-old female, was diagnosed with B-cell ALL. Cytogenetic analyses were normal, and real-time quantitative reverse transcriptase-polymerase chain reaction (RT-qPCR) testing for BCR-ABL was negative. The patient achieved complete remission (CR) after treatment with hyper CVAD (cyclophosphamide, vincristine, doxorubicin, dexamethasone) and alternating high-dose methotrexate and cytarabine chemotherapy. In addition, the patient underwent allogeneic HSCT from a fully human leukocyte antigen-matched sibling. Neither graft-versus-host disease (GVHD) nor infection developed during the follow-up period. One year after allogeneic HSCT, the patient complained of a painless, palpable nodule in the left breast. Ultrasonography revealed a $2.74 \times 2.69 \times 2.49 \mathrm{~cm}$ lobulated hetero-echogenic mass in the left breast (Fig. 1A), and positron emission tomography demonstrated a strong fluorodeoxyglucose uptake (Fig. ${ }_{1 B}$ and $\left.{ }_{1} C\right)$. Gun biopsy was performed, and an infiltration of primitive cells with focal terminal deoxynucleotidyl transferase-negative and CD34-positive 

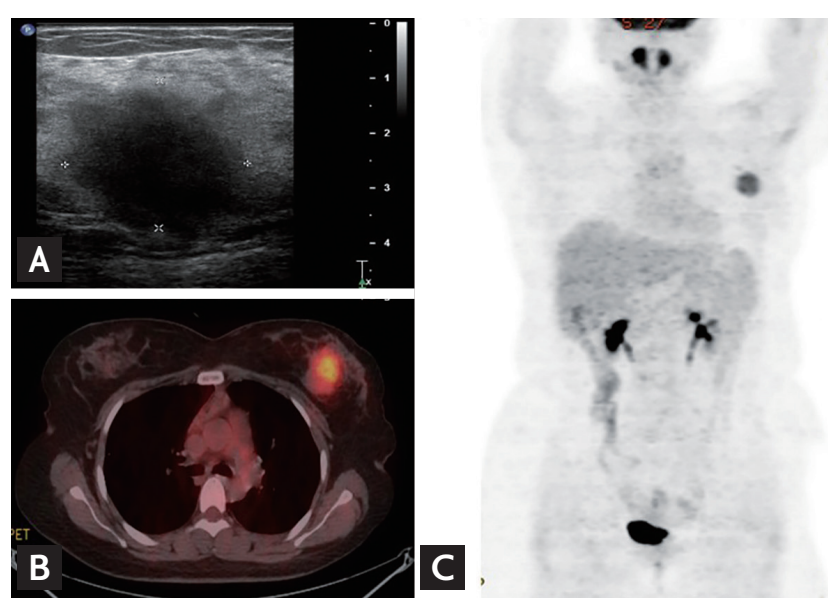

Figure 1. (A) Ultrasonography revealed a $2.7 \times 2.7 \mathrm{~cm}$ hetero-echogenic lobulated mass on the left breast. (B) Fusion imaging and $(\mathrm{C})$ maximum intensity projection imaging of positron emission tomography revealed an ill-defined ovalshaped mass with intense fluorodeoxyglucose uptake in the left breast.

cells was observed on histological examination, suggesting EMR of ALL (Fig. 2). However, short tandem repeat PCR of bone marrow aspirates showed complete chimerism. No leukemic lesions were identified in other sites such as the bone marrow or cerebrospinal fluid. The patient was treated with localized radiotherapy (5,000 cGy) beginning 410 days after allogeneic HSCT, followed by chemotherapy (daunorubicin, vincristine, prednisone, and L-asparaginase) on day 463 . We reexamined her chimerism status using a peripheral blood sample following radiotherapy and chemotherapy, and found that complete chimerism was maintained. After chemotherapy, thrombocytopenia did not improve, and on day 557, intracranial hemorrhage developed. The patient died on day 562 (Fig. 3).

There have been three previous reports of EMR of ALL in the breast after allogeneic HSCT [2-4]. The first case was a 15-year-old girl who received allogeneic HSCT after a second CR, and was diagnosed with EMR of the breast on day 246. She received radiotherapy and mild chemotherapy (Spiers's schedule) resulting in rapid disappearance of the breast mass [2]. As the patient and her family refused secondary allogeneic HSCT, she received only 6-mercaptopurine, methotrexate, and vincristine (1.5 mg intravenous injection every second month), and maintained CR for 10 months until isolated central nervous system (CNS) relapse [2]. The second case was also
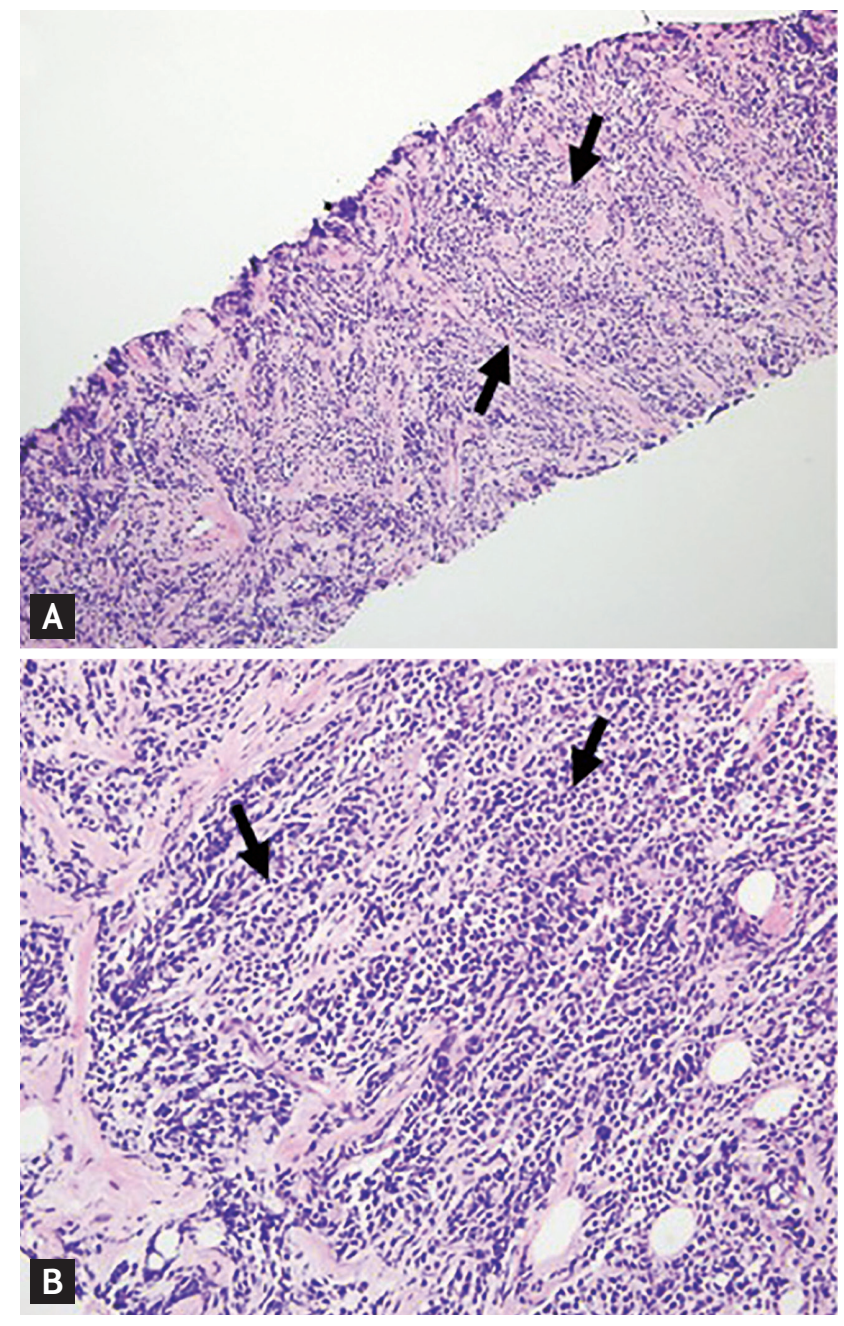

Figure 2. Histologic examination of the breast. Diffuse infiltration of primitive cells into the breast parenchyma and adjacent soft tissue was noted between arrows (A: H\&E, $\times 40$; B: $\mathrm{H} \& \mathrm{E}, \times 200)$.

a 15-year-old girl who received allogeneic HSCT after the first CR. She was diagnosed with EMR of the right breast, and received reinduction chemotherapy without radiotherapy. Four years later, she presented with recurrent masses in the right breast. She received remission induction therapy with local radiotherapy of the breast [3]. The third case was a 50-year-old female diagnosed with Philadelphia chromosome-positive ALL. EMR occurred in the right breast, left eyelid, left forehead and left lateral side of the neck after a second allogeneic HSCT during active hepatic GVHD [4].

In these three cases, the exact patient chimerism status was unclear or unavailable. However, allogeneic immune cells did not seem to effectively prevent relapse 


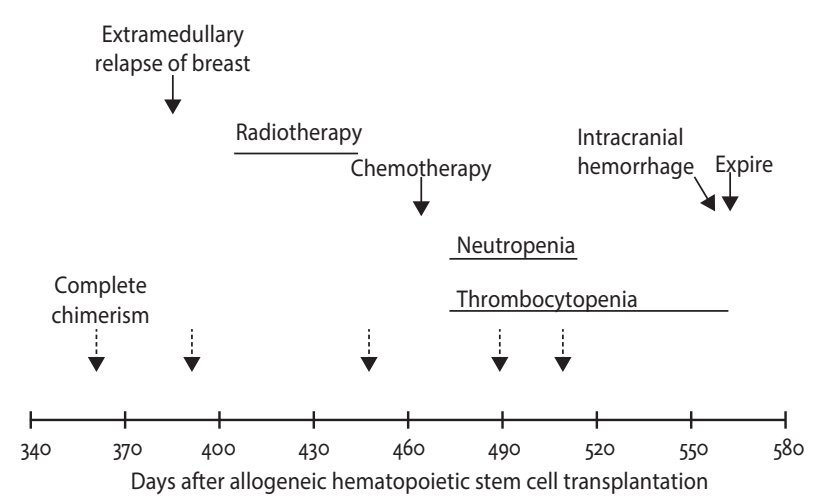

Figure 3. Important clinical events after allogeneic hematopoietic stem cell transplantation were depicted according to time course. Complete chimerism were maintained until day 509 (broken arrow).

in these cases, which was also observed in our own case. Isolated EMR of the breast may involve a different mechanism from bone marrow relapse or isolated EMR of the CNS or testes, and cannot be explained by an anatomical barrier preventing exposure to cytotoxic chemotherapy. Firstly, EMR has been reported more frequently in acute leukemia after allogeneic HSCT than after chemotherapy alone [1], in sites other than sanctuary sites such as the CNS or testes, including in the kidney, bone, intestine, and breast, although this is rare. Secondly, the median time from allogeneic HSCT to relapse is significantly longer for EMR than for EMR with bone marrow involvement or bone marrow relapse alone [5]. Thirdly, EMR-free survival is similar between patients with and without GVHD, which has an anti-leukemic effect [5].

In terms of treatment, radiotherapy may play a key role in EMR of the breast, as it does in EMR of sanctuary sites. In one case, a patient maintained CR after radiotherapy without high-dose chemotherapy before developing EMR in a sanctuary site [2], and another patient that did not receive radiotherapy experienced recurrent EMR at the same site in the breast [3]. Our patient also received radiotherapy after diagnosis of isolated EMR, and the mass disappeared with no evidence of leukemia relapse. The appropriate next treatment step in cases such as these remains unclear. Chemotherapy administered immediately after the disappearance of the breast mass, while the patient is in complete chimerism, or delayed chemotherapy may be options; however, the appropriate chemotherapy regimen and intensity for such cases remain to be investigated. The treatment sequence of chemotherapy and radiotherapy may also affect patient outcomes in EMR of the breast. Unfortunately, little is known about the optimal intensity, sequence, or timing of chemotherapy and radiotherapy in cases such as these. Thus, further studies are warranted in order to clarify the underlying mechanism and to determine the optimal treatment for isolated breast EMR, particularly in patients who maintain complete chimerism.

Keywords: Acute lymphoblastic leukemia; Extramedullary relapse; Breast

\section{Conflict of interest}

No potential conflict of interest relevant to this article was reported.

\section{REFERENCES}

1. Terwey TH, Hemmati PG, Nagy M, et al. Comparison of chimerism and minimal residual disease monitoring for relapse prediction after allogeneic stem cell transplantation for adult acute lymphoblastic leukemia. Biol Blood Marrow Transplant 2014;20:1522-1529.

2. Conter V, D'Angelo P, Rovelli A, et al. Isolated breast relapse after allogeneic bone marrow transplantation for childhood acute lymphoblastic leukemia. Med Pediatr Oncol 1992;20:165-168.

3. Savasan S, Abella E, Karanes C, Ravindranath Y. Recurrent breast relapses in a patient with acute lymphoblastic leukaemia following allogeneic bone marrow transplantation. Acta Haematol 1998;99:95-97.

4. Koca E, Goker H, Guven GS, et al. Unusual extramedullary recurrences and breast relapse despite hepatic GVHD after allografting in Ph+-ALL. Hematology 2006;11:105107.

5. Lee JH, Choi SJ, Lee JH, et al. Anti-leukemic effect of graft-versus-host disease on bone marrow and extramedullary relapses in acute leukemia. Haematologica 2005;90:1380-1388. 